\title{
PERIODS OF ITERATED INTEGRALS OF HOLOMORPHIC FORMS ON A COMPACT RIEMANN SURFACE
}

BY

\author{
SHU-YIN HWANG-MA
}

\begin{abstract}
Holomorphic forms are integrated iteratedly along paths in a compact Riemann surface $M$ of genus $g$, thus inducing a homomorphism from the fundamental group $\Gamma=\pi_{1}\left(M, P_{0}\right)$ to a proper multiplicative subgroup $G$ of the group of units in $\widehat{T\left(\Omega^{1^{*}}\right)}$, where $\Omega^{1}$ denotes the space of holomorphic forms on $M, \Omega^{1^{*}}$ is the complex dual of $\Omega^{1}, T$ means the associated tensor algebra and "' means completion with respect to the natural grading. The associated homomorphisms from $\Gamma / \Gamma^{(n+1)}$ to $G / G^{(n+1)}$ reduces to the classical case $H_{1}(M) \rightarrow \Omega^{1^{*}}$ when $n=1$. We show that the images of $\Gamma / \Gamma^{(n+1)}$ are always cocompact in $G / G^{(n+1)}$ and are discrete for all $n>2$ if and only if the Jacobian variety $J(M)$ of $M$ is isogenous to $E^{8}$ for some elliptic curve $E$ with complex multiplication.
\end{abstract}

The point of view taken here is suggested by Parsin's paper [4], although that paper does not contain any result beyond the construction of a homomorphism. Also the cocompactness of the image of $\Gamma / \Gamma^{(n+1)}$ in $G / G^{(n+1)}$ follows from general results of Sullivan [8] and Chen on a similar setting for "real" differentiable manifolds and "real" nilpotent groups; however we give a different proof.

1. Iterated integrals. All maps and forms are assumed to be complex analytic here; tensor products are taken over $\mathbf{C}$ and linear means complex linear.

Let $T=T\left(\Omega^{*}\right)$ and $T_{0}$ be the group of units in $T$. We will write elements in $T$ as formal infinite series $l_{0}+l_{1}+l_{2}+\cdots$ with $l_{j} \in \otimes^{j} \Omega^{1^{*}}$; then $T_{0}$ consists of elements with leading coefficient 1 .

Definition. An element $l=\Sigma l_{n} \in T$ is called a Lie element if $l_{n}=0$ and $l_{n}=$ combination of $\left[X_{i_{1}}, \ldots, X_{i_{n}}\right]$ 's where $\left\{X_{1}, \ldots, X_{g}\right\}$ is a base for $\Omega^{1^{*}}$ and

$$
\left[X_{i_{1}}, X_{i_{2}}\right]=X_{i_{1}} \otimes X_{i_{2}}-X_{i_{2}} \otimes X_{i_{1}}, \quad\left[X_{i_{1}}, \ldots, X_{i_{n}}\right]=\left[\left[X_{i_{1}}, \ldots, X_{i_{n-1}}\right], X_{i_{n}}\right] .
$$

Chen [1] proved that the formal power series

$$
\log \left(1+\sum_{n>0} \sum \alpha\left(i_{1}, \ldots, i_{n}\right) X_{i_{1}} \otimes \cdots \otimes X_{i_{n}}\right)
$$

is a Lie element if the coefficients $\alpha\left(i_{1}, \ldots, i_{n}\right)$ are given by successive integration along a path.

$G=\{l \in T \mid \log (l)$ is a Lie element $\}$ is a subgroup of $T_{0}$. We note that the filtration $\left\{G^{(n)}\right\}$ is induced by the pullback of the natural filtration on $T$ under the

Received by the editors November 16, 1979 and, in revised form, March 11, 1980.

1980 Mathematics Subject Classification. Primary 32G20; Secondary 14H05.

Key words and phrases. Iterated integrals, compact Riemann surfaces, period matrices, Jacobian varieties, Lie group, holomorphic forms. 
$\log$ map. Setting $\mathscr{F}=$ the free Lie algebra generated by $\Omega^{1^{*}}, \hat{\mathscr{F}}$ is the set of all Lie elements in $T$. We see that $\hat{\mathscr{F}}_{n} / \hat{\mathscr{F}}_{n+1} \cong G^{(n)} / G^{(n+1)}$ and

$$
\hat{\mathscr{F}}_{n} / \hat{\mathscr{F}}_{n+1} \cong\left[\left[\ldots\left[\Omega^{1^{*}}, \Omega^{1^{*}}\right], \Omega^{1^{*}}\right], \ldots, \Omega^{1^{*}}\right] \quad(n \text { times }) .
$$

$\left(\left\{\hat{\mathscr{F}}_{n}\right\}\right.$ is the natural grading of $\left.\hat{\mathscr{F}}_{\text {. }}\right)$

Let $\gamma \in \Gamma$ and associate with it a collection of $n$-linear functionals $\phi_{n}$ of holomorphic 1-forms as follows:

$$
\begin{gathered}
\phi_{0}(\gamma)=1, \quad \phi_{1}(\gamma)(\omega)=\int_{\gamma} \omega=\int \gamma^{*} \omega \\
\phi_{n}(\gamma)\left(\omega_{1}, \ldots, \omega_{n}\right)=\int_{S_{n}}\left(\gamma^{n}\right)^{*}\left(\omega_{1} \otimes \cdots \otimes \omega_{n}\right)=\int_{\gamma} \omega_{1} \otimes \cdots \otimes \omega_{n}
\end{gathered}
$$

where $n>0$ and $S_{n}=\left\{\left(t_{1}, \ldots, t_{n}\right) \in I^{n} \mid 0 \leqslant t_{1} \leqslant \cdots \leqslant t_{n} \leqslant 1\right\}$. Each $\phi_{n}(\gamma)$ can be regarded as an element of $\otimes^{n}\left(\Omega^{1^{*}}\right)$. Then we get a homomorphism $\rho$ from $\Gamma$ to $T_{0}$ induced by the $\phi_{i}$ 's (Parsin [4]). As in Ree [7], one can show that $\rho(\gamma) \in G$ for all $\gamma \in \Gamma$. Thus we arrive at a group homomorphism $\rho: \Gamma \rightarrow G$ where $G / G^{(2)}$ $=\Omega^{1^{*}}$.

2. Main results. We will choose on $M$ once and for all $g$ pairs of crosscuts $A_{1}, B_{1}, \ldots, A_{g}, B_{g}$ affording a canonical dissection of $M$. Then $\Gamma=\pi_{1}\left(M, P_{0}\right)=$ $\left\langle A_{1}, \ldots, A_{g}, B_{1}, \ldots, B_{g} \mid \Pi_{j=1}^{g}\left[A_{j}, B_{j}\right]=1\right\rangle$. And let $\omega_{1}, \ldots, \omega_{g}$ be the normalized base of $\Omega^{1}$ w.r.t. $A_{1}, \ldots, A_{g}, B_{1}, \ldots, B_{g} ; X_{1}, \ldots, X_{g}$ be the base for $\Omega^{1^{*}}$ dual to $\omega_{1}, \ldots, \omega_{g} . Z$ will denote the normalized period matrix $\left(z_{i j}\right)$ where $z_{i j}=\int_{B_{i}} \omega_{j}$.

Define $\omega[i]=\omega_{i}$ and

$$
\omega\left[i_{1}, \ldots, i_{n}\right]=\omega\left[i_{1}, \ldots, i_{n-1}\right] \otimes \omega\left[i_{n}\right]-\omega\left[i_{2}, \ldots, i_{n}\right] \otimes \omega\left[i_{1}\right]
$$

By a direct computation (or Ree [7]), one sees

$$
\left[X_{i_{1}}, \ldots, X_{i_{n}}\right]\left(\omega_{j_{1}} \otimes \cdots \otimes \omega_{j_{n}}\right)=\left(X_{i_{1}} \otimes \cdots \otimes X_{i_{n}}\right)\left(\omega\left[j_{1}, \ldots, j_{n}\right]\right)
$$

and

$$
\left[X_{i_{1}}, \ldots, X_{i_{n}}\right]\left(\omega\left[j_{1}, \ldots, j_{n}\right]\right)=n\left(X_{i_{1}} \otimes \cdots \otimes X_{i_{n}}\right)\left(\omega\left[j_{1}, \ldots, j_{n}\right]\right) .
$$

So we can identify $G^{(n)} / G^{(n+1)}$ with $\left\langle\omega\left[j_{1}, \ldots, j_{n}\right] \mid\left(j_{1}, \ldots, j_{n}\right) \in I\right\rangle^{*}$ where $I=$ $\{1, \ldots, g\}^{n}$.

We define $\left[\gamma_{1}, \ldots, \gamma_{n}\right]=\left[\left[\ldots\left[\gamma_{1}, \gamma_{2}\right], \gamma_{3}\right], \ldots, \gamma_{n}\right]$ for $\gamma_{1}, \ldots, \gamma_{n} \in \Gamma$ and observe that

$\rho\left(\left[A_{j_{1}}, \ldots, A_{j_{n}}\right]\right) \equiv\left[X_{j_{1}}, \ldots, X_{j_{n}}\right] \bmod$
Further $\rho\left(\left[A_{j_{1}}, \ldots, A_{j_{n-1}}, B_{j_{n}}\right]\right) \equiv \sum_{k} z_{j_{n} k}\left[X_{j_{1}}, \ldots, X_{j_{n-1}}, X_{k}\right]$.

Let $I^{\prime}$ be a subset of $I$ such that the monomials

$$
\left[X_{i_{1}}, \ldots, X_{i_{n}}\right]_{\left(i_{1}, \ldots, i_{n}\right) \in I^{\prime}}
$$

form a (complex) basis of the vector space of homogeneous Lie elements of degree $n$; that is, the space $G^{(n)} / G^{(n+1)}$. Then since the imaginary part of the period matrix $\left(z_{i j}\right)$ is invertible, the set

$$
\left[X_{i_{1}}, \ldots, \sum_{k} z_{i_{n} k} X_{k}\right]_{\left(i_{1}, \ldots, i_{n}\right) \in I^{\prime}}
$$


is also a complex basis, and the sets (1) and (2), taken together, form a basis of the real vector space $G^{(n)} / G^{(n+1)}$.

Proposition 2.1. $\rho\left(\Gamma^{(n)}\right)$ is cocompact in $G^{(n)} / G^{(n+1)}$.

If $Z=\left(z_{i j}\right)$ has entries in $Z(\sqrt{-\alpha})$ for some $\alpha \in \mathbf{N}$, then it is trivial that $\rho\left(\Gamma^{(n)}\right)$ is discrete in

$$
G^{(n)} / G^{(n+1)} \text {. }
$$

But since the image of $\Gamma^{(n)}$ in (3) is always finitely generated, an argument using a common denominator of the generators allows the same conclusion whenever the entries of $Z$ lie in $\mathbf{Q}(\sqrt{-\alpha})$. So we have

Proposition 2.2. If $Z \in M_{g}(\mathbf{Q}(\sqrt{-\alpha}))$ for some $\alpha \in \mathbf{N}$, then the image of $\Gamma^{(n)}$ is discrete in $G^{(n)} / G^{(n+1)}$.

For the converse, we first show

Proposition 2.3. If the image of $\Gamma^{(2)}$ is discrete in $G^{(2)} / G^{(3)}$, then all $z_{i j} \in \mathbf{Q}(\sqrt{-\alpha})$ for some $\alpha \in \mathbf{N}$.

Proof. $G^{(2)} / G^{(3)} \stackrel{\log }{\cong}\left[\Omega^{1^{*}}, \Omega^{1^{*}}\right] \cong\left[\Omega^{1}, \Omega^{1}\right]^{*}$. By the proof of Proposition 2.1 and the discreteness of $\rho\left(\Gamma^{(2)}\right)$ in $G^{(2)} / G^{(3)}$, every element in the image of $\Gamma^{(2)}$ must be a rational combination of

$$
\rho\left(\left[A_{i}, A_{j}\right]\right) \equiv \Delta_{i j} \quad \text { and } \quad \rho\left(\left[A_{i}, B_{j}\right]\right) \equiv S_{i j} \bmod G^{(3)}, \quad 1 \leqslant j<i \leqslant g .
$$

Write $\rho\left(\left[A_{1}, B_{k}\right]\right) \equiv \Sigma_{i>j} \lambda_{i j}^{k} \Delta_{i j}+\sum_{i>j} \mu_{i j}^{k} S_{i j}$ where $1 \leqslant k<g, \lambda_{i j}^{k}, \mu_{i j}^{k} \in \mathbf{Q}$.

Taking the value at $\left[\omega_{1}, \omega_{n}\right], 1<n \leqslant g$ :

$$
\begin{aligned}
& z_{k n}=-\lambda_{n 1}^{k}-\sum_{j<n} \mu_{n j}^{k} z_{j 1} ; \\
& z_{12}=-\lambda_{21}^{1}-\mu_{21}^{1} z_{11}, \quad(k, n)=(1,2) ; \\
& z_{1 n}=-\lambda_{n 1}^{1}-\sum_{j<n} \mu_{n j}^{1} z_{j 1}, \quad(k, n)=(1, n) ;
\end{aligned}
$$

inductively, we see $z_{1 n}$ is a rational combination of 1 and $z_{11}$ for $n>1$; (2.3.1) then shows $z_{k n}$ is a rational combination of 1 and $z_{11}$. Therefore, all $z_{i j} \in \mathbf{Q}+\mathbf{Q} z_{11}$. $Z=A+B z_{11}$ with $A$ and $B$ in $M_{g}(\mathbf{Q})$.

Write $z_{11}=x_{11}+\sqrt{-1} y_{11}$ with $x_{11}, y_{11} \in \mathbf{R}$ and $y_{11}>0$;

$$
\rho\left(\left[B_{1}, B_{k}\right]\right) \equiv \sum_{i>j}\left(c_{i j} \Delta_{i j}+d_{i j} S_{i j}\right), \quad c_{i j}, d_{i j} \in \mathbf{Q} ;
$$

taking value at $\left[\omega_{m}, \omega_{n}\right]$ :

$$
z_{1 m} z_{k n}-z_{k m} z_{1 n}=-c_{n m}+\sum_{j<m} d_{m j} z_{j n}-\sum_{j<n} d_{n j} z_{j m}
$$

is a rational combination of 1 and $z_{11}$.

Let $(m, n)=(1,2),(1, k)=(1,2)$; we have

$$
z_{11}\left(a_{22}+b_{22} z_{11}\right)-\left(a_{12}+b_{12} z_{11}\right)^{2}=a+b z_{11}
$$


where $a_{i j}, b_{i j}, a, b \in \mathbf{Q}$. So

$$
\begin{aligned}
& a+b z_{11}=-a_{12}^{2}+\left(a_{22}-2 a_{12} b_{12}\right) z_{11}+\left(b_{22}-b_{12}^{2}\right) z_{11}^{2}, \\
& b_{22}-b_{12}^{2}=\frac{1}{y_{11}^{2}} \operatorname{det}\left(\begin{array}{ll}
y_{11} & y_{12} \\
y_{21} & y_{22}
\end{array}\right)>0
\end{aligned}
$$

Thus $z_{11}$ satisfies a quadratic equation over $\mathbf{Q}, z_{11}=x+y \sqrt{-\alpha}$ for some $x$ and $y$ in $\mathbf{Q}, \alpha \in \mathbf{N}$ and $y>0$. And hence $z_{i j} \in \mathbf{Q}(\sqrt{-\alpha})$ for all $i, j=1,2, \ldots, g$. Q.E.D.

Proposition 2.4. $\rho(\Gamma)$ is a lattice in $G / G^{(n+1)}$ for all $n \geqslant 2$ if and only if the image of $\Gamma^{(n)}$ is a lattice in $G^{(n)} / G^{(n+1)}$ for all $n \geqslant 2$.

Proof. If $\rho\left(\Gamma^{(n)}\right)$ is a lattice in $G^{(n)} / G^{(n+1)}$ for all $n \geqslant 2$, then the cocompactness of the image of $\Gamma$ in $G / G^{(n+1)}$ follows by induction from the fact that

$$
G /\left(G^{(n+1)} \cdot \Gamma\right) \rightarrow G /\left(G^{(n)} \cdot \Gamma\right)
$$

is a fibration with compact fibre.

To show the discreteness of $\rho(\Gamma)$ in $G / G^{(n+1)}$ we define $H$ to be $G^{(n)} / G^{(n+1)} \cap$ Image of $\Gamma$ and note that $H$ is closed. We first show that $H$ is discrete in $G^{(n)} / G^{(n+1)}$, hence in $G / G^{(n+1)}$.

$G^{(n)} / G^{(n+1)}$ is a vector space over $\mathbf{C}$ and $\rho\left(\Gamma^{(n)}\right)$ is discrete and cocompact in $G^{(n)} / G^{(n+1)}, H \supset \rho\left(\Gamma^{(n)}\right)$ contains $2 \times \operatorname{dim}_{C} G^{(n)} / G^{(n+1)}$ linearly independent elements over $\mathbf{R}$, say, $a_{1}, \ldots, a_{s}$. It suffices to show every element in $H$ is a rational combination of $a_{1}, \ldots, a_{s}$.

Assuming the negation, we can find an element $a \in H, a=\sum_{j=1}^{t} \lambda_{j} a_{i j}$ where $i_{1}, \ldots, i_{t} \in\{1, \ldots, s\}$ and distinct $\lambda_{1}, \ldots, \lambda_{t} \in \mathbf{R} \backslash \mathbf{Q}$. Then, for any $c \in[0,1]$, there are sequences of integers $n_{j}, m_{j}^{k}, j=1,2, \ldots, k=1, \ldots, t$, such that

$$
n_{j} a+\sum_{k=1}^{t} m_{j}^{k} a_{i_{k}} \rightarrow c a_{i_{1}}+a^{\prime} \quad \text { as } j \rightarrow \infty
$$

where $a^{\prime}$ is a real combination of $a_{i_{2}}, \ldots, a_{i_{1}}$. Because $H$ is closed, $c a_{i_{1}}+a^{\prime} \in H$ and $H$ contains uncountably many elements. But $H \subseteq \rho\left(\Gamma / \Gamma^{(n+1)}\right)$ where $\Gamma / \Gamma^{(n+1)}$ is a finitely generated nilpotent group, hence countable. We conclude that $H$ is discrete.

Now to show $\rho(\Gamma)$ is discrete in $G / G^{(n+1)}$, we let $b_{0} \in \rho\left(\Gamma / \Gamma^{(n+1)}\right)$ and image of $b_{0}$ in $\rho\left(\Gamma / \Gamma^{(n)}\right)$ be $c_{0}$. There is an open neighborhood $V$ of $c_{0}$ in $G / G^{(n)}$ such that $V \cap \rho\left(\Gamma / \Gamma^{(n)}\right)=\left\{c_{0}\right\}$. If $b \in \rho\left(\Gamma / \Gamma^{(n+1)}\right) \cap$ preimage of $V$ in $G / G^{(n+1)}$, then $b^{-1} b_{0} \in H$. Since $H$ is discrete, there is an open neighborhood $U$ of the identity $e$ in $G / G^{(n+1)}$ such that $U \cap \rho\left(\Gamma^{(n)} / \Gamma^{(n+1)}\right)=\{e\} . b_{0} U \cap$ preimage of $V$ is then an open neighborhood of $b_{0}$ in $G / G^{(n+1)}$ and contains no other elements of $\rho\left(\Gamma / \Gamma^{(n+1)}\right)$. Therefore $\rho\left(\Gamma / \Gamma^{(n+1)}\right)$ is discrete.

Conversely, assume $\rho(\Gamma)$ is a lattice in $G / G^{(n+1)}$ for all $n \geqslant 2$; we have to show $\rho\left(\Gamma^{(n)}\right)$ is a lattice in $G^{(n)} / G^{(n+1)}$ for all $n \geqslant 2$. In view of Proposition 2.1 we only have to show the discreteness, but this is obvious. Q.E.D.

So we have proved the main theorem: 
THEOREM 2.5. The image of $\Gamma$ under $\rho$ is a lattice in $G / G^{(n+1)}$ for all $n \geqslant 2$ if and only if $Z \in M_{g}(K)$ for some imaginary quadratic field $K$.

The condition in the prior theorem can be interpreted as a condition on the Jacobian $J(M)$ by the following

Proposition 2.6. $Z \in M_{g}(K)$ for some imaginary quadratic field $K$ if and only if $J(M)$ is isogenous to $E^{g}$ where $E$ is an elliptic curve with complex multiplication by an order in $K$.

The proof of this proposition is nothing more than an easy checking of the definitions.

3. Examples. Fist we remark that the condition in Theorem 2.5 is neither null nor redundant. To be more precise, the Klein surface given by the equation $x^{3} y+y^{3}+$ $x=0$ has a normalized period matrix

$$
Z=\left(\begin{array}{ccc}
-\frac{1}{8}+\frac{3 \sqrt{7}}{8} i & -\frac{1}{4}-\frac{\sqrt{7}}{4} i & -\frac{3}{8}+\frac{\sqrt{7}}{8} i \\
-\frac{1}{4}-\frac{\sqrt{7}}{8} i & \frac{1}{2}+\frac{\sqrt{7}}{2} i & -\frac{1}{4}-\frac{\sqrt{7}}{4} i \\
-\frac{3}{8}+\frac{\sqrt{7}}{8} i & -\frac{1}{4}-\frac{\sqrt{7}}{4} i & \frac{7}{8}+\frac{3 \sqrt{7}}{8} i
\end{array}\right)
$$

(Rauch and Lewittes [6, pp. 297-308]) where one sees that all $z_{i j} \in \mathbf{Q}(\sqrt{-7})$; while the Riemann surface of $y^{2}=x^{6}-x$ has a normalized period matrix

$$
Z=\left(\begin{array}{cc}
\frac{1-\sqrt{5}+i \sqrt{10+2 \sqrt{5}}}{4} & \frac{2-\sqrt{5}+i \sqrt{10-2 \sqrt{5}}}{4} \\
\frac{3-\sqrt{5}+i \sqrt{10-2 \sqrt{5}}}{4} & \frac{i \sqrt{10-2 \sqrt{5}}}{2}
\end{array}\right)
$$

(Rauch [5, pp. 105-108, 155-159]) where $Z \notin M_{2}(K)$ for any imaginary quadratic field $K$.

By the results in Koblitz [3], we are able to list all the Fermat curves whose Jacobians are isogenous to $E^{g}$ for some elliptic curve $E$ with $C M$.

(3.1) The Jacobian $J_{d}$ of the Fermat curve $x^{d}+y^{d}=1$ is isogenous to $E^{g}$ where $g=(d-1)(d-2) / 2$ for some elliptic curve $E$ with complex multiplication if and only if $d=3,4$ or 6 .

\section{REFERENCES}

1. K. T. Chen, Integration of paths, geometric invariants and a generalized Baker-Hausdorff formula, Ann. of Math. (2) 65 (1957), 163-178.

2. __ Algebras of iterated path integrals and fundamental groups, Trans. Amer. Math. Soc. 156 (1971), 359-379.

3. N. Koblitz, Gamma function identities and elliptic differentials on Fermat curves, Duke Math. J. 45 (1978), 87-99.

4. A. N. Parsin, A generalization of the Jacobian variety, Amer. Math. Soc. Transl. (2) 84 (1969), 187-196. 
5. H. E. Rauch, Theta functions with applications to Riemann surfaces, William \& Wilkins, Baltimore, Md., 1974.

6. H. E. Rauch and J. Lewittes, The Riemann surface of Klein with 168 automorphisms, Problems in Analysis (Papers dedicated to Solomon Bochner, 1969), Princeton Univ. Press, Princeton, N.J., 1970, pp. 297-308.

7. R. Ree, Lie elements and an algebra associated with shuffles, Ann. of Math. (2) 68 (1958), 210-220.

8. D. Sullivan, Differential forms and the topology of manifolds, Manifolds-Tokyo 1973 (Proc. Internat. Conf. on Manifolds and Related Topics in Topology), Univ. Tokyo Press, Tokyo, 1975, pp. 37-49.

Institute of Mathematics, Academia Sinica, Nankang, Taipei, Taiwan 